\title{
Neurotrophic keratitis: current challenges and future prospects
}

This article was published in the following Dove Press journal:

Eye and Brain

\section{Piera Versura \\ Giuseppe Giannaccare \\ Marco Pellegrini \\ Stefano Sebastiani \\ Emilio C Campos}

Ophthalmology Unit, Department of Experimental, Diagnostic and Specialty Medicine (DIMES), Alma Mater Studiorum University of Bologna, Sant'Orsola-Malpighi Teaching Hospital, Bologna, Italy
Correspondence: Piera Versura Ophthalmology Unit, Department of Experimental, Diagnostic and Specialty Medicine (DIMES), Alma Mater Studiorum University of Bologna, Sant'Orsola-Malpighi Teaching Hospital, Via Pelagio Palagi 9, 40I38 Bologna, Italy Tel +39 05। $2 \mid 42850$

$\mathrm{Fax}+390516362846$

Email piera.versura@unibo.it
Abstract: Neurotrophic keratitis (NK) is a degenerative corneal disease caused by damage of trigeminal corneal innervation, which leads to spontaneous epithelial breakdown and corneal ulceration. The impairment of corneal sensory innervation causes the reduction of both protective reflexes and trophic neuromodulators that are essential for the vitality, metabolism, and wound healing of ocular surface tissues. A wide range of ocular and systemic conditions, including herpetic keratitis, ocular chemical burns, corneal surgery, diabetes, multiple sclerosis, and neurosurgical procedures, can cause NK by damaging trigeminal innervation. Diagnosis of NK requires careful investigation of any ocular and systemic condition associated with the disease, complete ocular surface examination, and quantitative measurement of corneal sensitivity. The clinical stages of NK range from corneal epithelial alterations (stage 1) to persistent epithelial defect (stage 2) and ulcer (stage 3), which may progress to corneal perforation. Management of NK is based on clinical severity, and the aim of the therapy is to halt the progression of corneal damage and promote epithelial healing. Although several medical and surgical treatments have been proposed, no therapies are currently available to restore corneal sensitivity, and thus, NK remains difficult and challenging to treat. The purpose of this review is to summarize available evidence on the pathogenesis, diagnosis, and treatment of NK. Novel medical and surgical therapies including the topical administration of nerve growth factor and corneal neurotization are also described.

Keywords: neurotrophic keratitis, neurotrophic corneal ulcer, corneal nerves

\section{Introduction}

Neurotrophic keratitis (NK) is a rare degenerative corneal disease caused by damage of trigeminal innervation. Corneal nerves play an essential role in tear production and preservation of the normal metabolism and function of the ocular surface. Loss of sensitivity impairs corneal wound healing, leading to epithelial changes including punctate epithelial keratopathy, persistent epithelial defects, and corneal ulcer. ${ }^{1}$

Various ocular and systemic diseases can cause damage to the fifth cranial nerve at different levels, from the trigeminal nucleus to the corneal nerve endings. Common causes are herpetic keratitis, diabetes, chemical or surgical damage, and neurosurgical procedures. ${ }^{2,3}$ Medical treatment consists mainly of supportive measures that do not address the underlying cause of the disease. Recently, novel promising treatments have been proposed and are currently under evaluation. These include mainly topical treatment with nerve growth factor (NGF) and surgical corneal neurotization. Such approaches target the pathogenic elements of NK, carrying the potential of restoring normal corneal innervation and sensitivity. 


\section{Pathogenesis}

The cornea is innervated by the ophthalmic branch of the trigeminal nerve as well as by autonomic nerves. Nerve bundles enter the cornea at the limbus, move toward the center below the anterior third of the stroma, penetrate Bowman's layer, and create a dense network of nerve fibers between Bowman's layer and basal epithelial cells (i.e., sub-basal nerve plexus). ${ }^{4}$ The great number of sensory nerve endings makes the cornea the most densely innervated tissue in the human body. ${ }^{5}$ Nerves have a key role in maintaining a healthy ocular surface, both by triggering protective reflexes after injury, and by providing trophic factors to the corneal cells. Corneal denervation causes decreased vitality, metabolism, and mitosis of epithelial cells, with subsequent epithelial changes including intracellular edema, loss of microvilli, and abnormal development of the basal lamina. ${ }^{6,7}$

Corneal sensory innervation reacts to mechanical, chemical, and thermal stimuli with two reflex arcs: a motor arc eliciting blinking, and an autonomous arc stimulating tear secretion. Therefore, the reduction of corneal sensation impairs these two reflexes with an alteration of production and stability of the tear film. ${ }^{8}$

Corneal nerves and epithelial cells support each other mutually through the release of trophic factors promoting epithelial cell proliferation, migration, and differentiation as well as nerve development and survival. Corneal nerves express many epitheliotrophic neuromediators, such as substance $\mathrm{P}$, calcitonin gene-related peptide, acetylcholine, noradrenaline, serotonin, neuropeptide $\mathrm{Y}$, and vasointestinal peptide. On the other side, corneal epithelial cells release various neurotrophic growth factors, including NGF, ciliary neurotrophic factor, and glial-cell-derived neurotrophic factor. These factors are fundamental in ocular surface homeostasis and wound healing. ${ }^{4}$

In particular, NGF is a neurotrophin discovered in the early 1950s by R. Levi-Montalcini that promotes neuronal sprouting by intact and injured neurons. It provides trophic support to neurons after injury and reverses pathologic changes induced by peripheral nerve damage. ${ }^{9}$ NGF exerts its biologic functions by binding to low-affinity p75NTR (neurotrophin receptor, a member of the tumor necrosis factor receptor superfamily) and high-affinity TrkA receptor (a tyrosine kinase receptor family). The human cornea produces NGF and expresses high-affinity NGF receptors. ${ }^{10}$ NGF demonstrated an important role in the integrity and function of the ocular surface, stimulating both epithelial and nerve fiber proliferation and survival. ${ }^{11}$ In vivo studies proved that NGF concentration increases after injury, and that administration of NGF accelerates corneal healing. ${ }^{10}$

These complex reciprocal relations between epithelial cells and nerves are crucial for the corneal physiologic renewal and wound healing. An impairment of corneal sensitivity interrupts these trophic relations and may produce the pathologic changes typical of NK.

\section{Causes}

All conditions that impair trigeminal innervation at any level can cause NK, such as ocular surface diseases, systemic diseases, and central or peripheral nervous damages (Figure 1)..$^{12,13}$

Herpes simplex and herpes zoster eye infections are among the most common conditions leading to corneal anesthesia. In a large case series from Bonini et al, herpetic keratitis was the most common cause of NK $(27 \%$ of the patients). ${ }^{2}$ A severe reduction of sub-basal nerve plexus density was demonstrated in herpetic keratitis. ${ }^{14}$

Corneal surgeries like laser-assisted in situ keratomileusis, keratoplasty, and corneal incisions for cataract may disrupt corneal innervation and cause NK. ${ }^{15,16}$ After nerve transection at the peripheral cornea, the distal nerves undergo Wallerian degeneration with immediate loss of sensation to the corresponding region. Nerve regeneration occurs gradually over months with variable return of corneal sensitivity. ${ }^{15}$ Other ocular surface conditions associated with NK are corneal chemical and traumatic injuries, corneal dystrophies, contact lens wearing, and chronic use of topical medications (such as anesthetics, beta-blockers, antivirals, glaucoma medications, antibiotics, and nonsteroidal anti-inflammatory drugs)..$^{12,13}$

Surgical procedures for the treatment of trigeminal neuralgia may induce permanent damage to the trigeminal nerve with subsequent corneal anesthesia. The main surgical options include microvascular decompression, balloon compression, radiofrequency thermocoagulation, and gamma knife radiosurgery. The intraoperative damage occurs at the preganglionic or ganglionic region of the nerve and is not associated with a reduction of sub-basal nerve plexus density. ${ }^{17}$ Other less common causes of trigeminal palsy are intracranial and orbital tumors, facial trauma, aneurysm, and stroke. ${ }^{1,12}$

Systemic conditions like diabetes, multiple sclerosis, and leprosy may impair corneal sensitivity and cause NK. In particular, neuropathy is one of the most common complications of diabetes mellitus and results from microvascular damage 


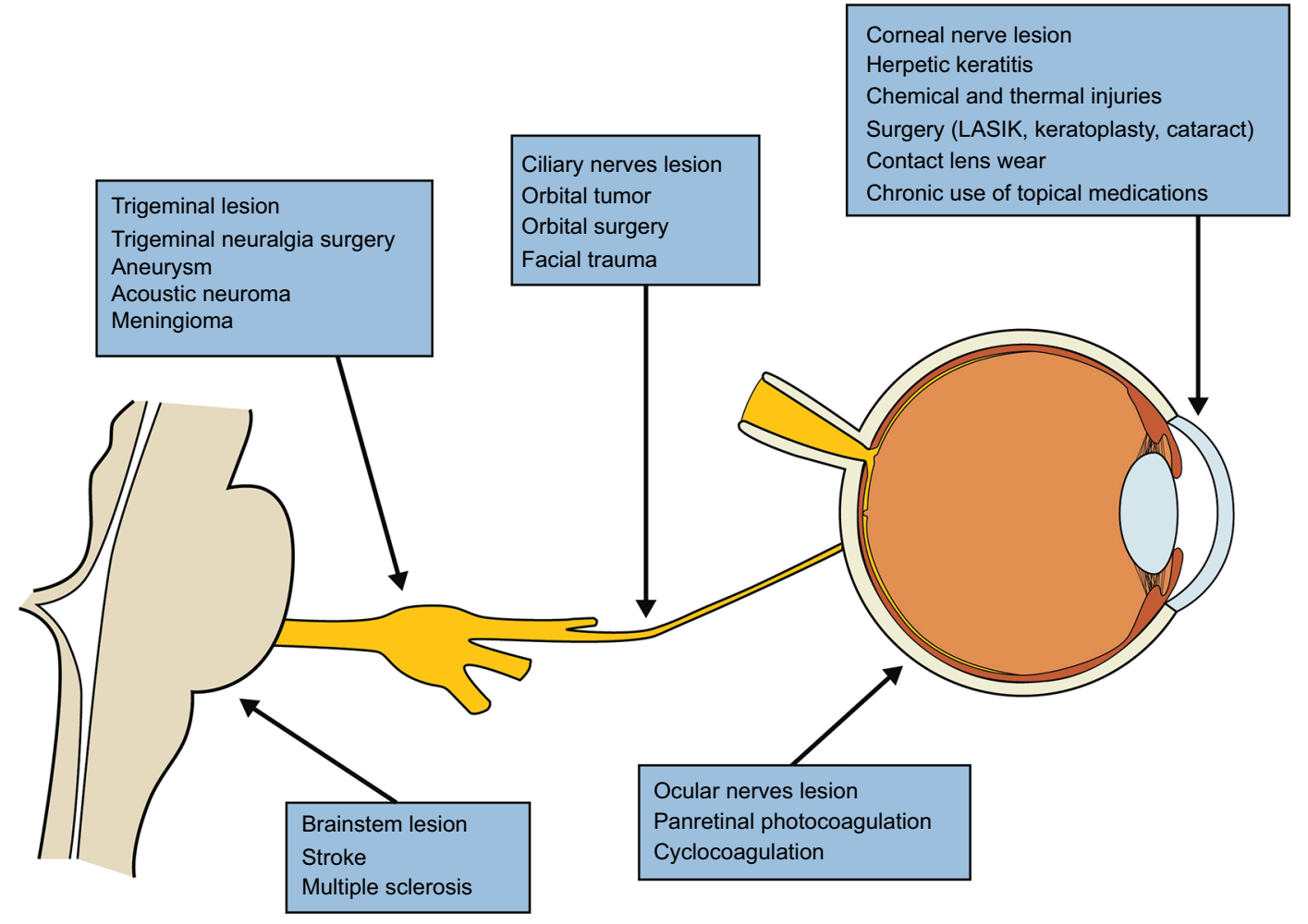

Figure I Common causes of neurotrophic keratitis.

Abbreviation: LASIK, laser-assisted in situ keratomileusis.

of myelinated nerve fibers. The extent of nerve damage has been demonstrated to be associated with the duration of hyperglycemia. ${ }^{18}$ Corneal nerve evaluation in patients with diabetes has shown that the decreased corneal sensitivity was related to a reduction of sub-basal nerve density, with abnormally tortuous nerve fiber bundles. Additionally, a correlation between the decrease of corneal sensitivity and the degree of somatic polyneuropathy was found. ${ }^{18}$ Recent studies using in vivo confocal microscopy (IVCM) have reported an association between levels of glycated hemoglobin and the density of corneal innervation. ${ }^{19}$

Long-term systemic therapy with antipsychotics and antihistamines may also cause NK. Very rare congenital causes of NK include Riley-Day syndrome, Moebius syndrome, Goldenhar-Gorlin syndrome, and congenital corneal anesthesia. ${ }^{12}$

\section{Clinical presentation}

The clinical presentation of NK is characterized by welldefined and progressive ocular surface changes, which are caused by the loss of sensitivity, regardless the cause of trigeminal damage. A three-stage classification of NK based on severity of corneal damage was introduced by Mackie. ${ }^{1}$
Stage 1 is characterized by corneal abnormalities such as punctate keratopathy, corneal edema, corneal epithelial hyperplasia, and irregularity (Figure 2A). Rose Bengal staining of the conjunctiva, increased tear viscosity, and decreased breakup time are often associated. In long-standing cases, stromal scarring and superficial corneal neovascularization may develop.

Stage 2 is characterized by a recurrent or persistent epithelial defect, commonly located paracentrally in the superior half of the cornea. The epithelial defect is often delimited by smooth, rolled, and loose edges of an opaque and edematous epithelium (Figure 2B). Stromal edema with Descemet's membrane folds may be observed and sometimes anterior chamber inflammation is associated.

Stage 3 is characterized by corneal ulcer (Figure 2C). The stromal melting may progress to corneal thinning and eventually perforation. This may occur without significant ocular symptoms because of impaired corneal sensitivity; however, patients may complain of blurred vision in case of corneal ulcer, edema, or scarring. ${ }^{1,12}$

\section{Diagnosis}

Patient's clinical history should be accurately collected and reviewed to identify any ocular or systemic disease potentially 

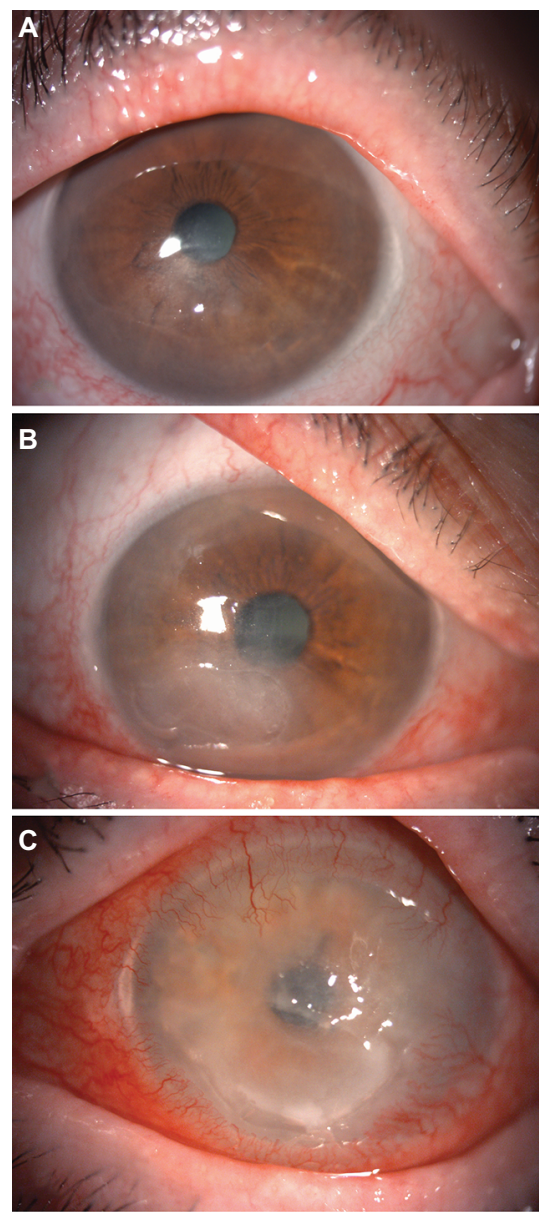

Figure 2 Three-stage classification of neurotrophic keratitis.

Note: Stage I: cloudy and irregular corneal epithelium (A); stage 2: large epithelial defect characterized by smooth edges (B); stage 3: deep corneal ulcer and stromal melting (C).

associated with the disorder, such as ocular surface conditions (e.g., previous herpetic infection, ocular surgery, chemical burns, topical drug use, contact lens wearing), systemic diseases (e.g., diabetes, multiple sclerosis), and neurologic conditions (e.g., brain tumors, neurosurgery, stroke, trauma).

A careful ocular surface examination should be performed. In particular, eyelids should be examined to rule out the presence of lagophthalmos or blepharitis. Slit-lamp examination is fundamental to detect sings of NK and to classify NK severity, ranging from punctate corneal keratopathy to stromal melting and corneal perforation. Corneal anesthesia causes lack of ocular discomfort symptoms; thus, NK should be always suspected in case of significant discrepancy between ocular signs and symptoms. Ocular examination helps to identify also possible causes of decreased corneal sensitivity: corneal scarring may indicate previous injuries or infections; patchy iris atrophy may be a sign of previous herpetic infection. Fundus examination may show signs of diabetic retinopathy or optic nerve abnormalities due to intracranial pathology. ${ }^{12,13}$

Vital staining with fluorescein or lissamine green is useful to assess corneal and conjunctival epithelial integrity. Tear film production and stability should be evaluated with lacrimal function tests. It is known that neurotropic keratitis is associated with an increase of tear osmolarity and a decrease of both Schirmer test and tear breakup time values. ${ }^{8,20}$

The decrease or absence of corneal sensitivity is the diagnostic hallmark of NK. Corneal sensitivity can be quantitatively measured by Cochet-Bonnet esthesiometer, which employs a nylon monofilament of adjustable length touching the cornea and recording the patient's response. Each quadrant of the cornea may be tested. As the length of the filament is decreased, the pressure transmitted is increased. ${ }^{21}$ The noncontact Belmonte esthesiometer is another device allowing to investigate also chemical and thermal corneal sensitivity. ${ }^{22}$ However, it is typically reserved for research purposes, and not routinely used in clinical practice to date.

IVCM is a noninvasive imaging technique that allows the in vivo study of the different layers of the ocular surface at a cellular level. IVCM has been used to evaluate corneal nerve morphology in healthy and diseased corneas. A significant reduction of corneal nerve density strongly correlated with the decrease of corneal sensation was shown in patients with NK. ${ }^{14-18}$ In addition, IVCM showed also a higher number of hyper-reflective keratocytes and a lower epithelial and endothelial cell density in patients with NK. ${ }^{16}$ These findings suggest that corneal sensory nerves play a role also in the function and survival of the corneal endothelium.

\section{Treatment}

Treatment approach of NK should be prompt and based on the stage and severity of the disease. Despite various medical and surgical therapies having been proposed, NK remains yet difficult and challenging to treat, and the lack of positive response is commonly observed in the clinical practice.

In stage 1 , the therapy aims at preventing an epithelial breakdown and improving epithelial quality. All topical and systemic medications associated with ocular surface toxicity should be discontinued. Preservative-free tear substitutes and lubricant ointments are useful to improve lubrication and ocular surface health. Other associated ocular surface diseases, such as dry eye, exposure keratitis, and limbal stem cell deficiency, should be properly treated. ${ }^{12}$

In stage 2, therapy is addressed to promote healing of the epithelial defect, and to prevent the progression to corneal ulcer. Patient should be monitored frequently, because the 
progression to stromal melting and perforation may occur without any symptom. Topical antibiotics are recommended in this stage to prevent infections. On the contrary, topical steroids should be used with caution, because they may inhibit the healing process and increase the risk of corneal melting. Therapeutic contact lenses are useful to promote corneal healing, maintaining a fluid layer in stable contact with the cornea, and protecting it from the rubbing of the eyelids. However, attention should be paid because of the increased risk of infections. ${ }^{23}$

Autologous serum eye drops have become increasingly popular for treating ocular surface disorders, including NK. ${ }^{24}$ Autologous serum contains several natural components of the normal tears, such as growths factors, neuromediators, cytokines, and vitamins. ${ }^{25}$ These substances are known to promote proliferation, migration, and differentiation of the ocular surface epithelium, and are essential in corneal homeostasis and wound healing. ${ }^{26}$ Matsumoto et al reported the complete healing of all the 14 eyes with NK treated with autologous serum drops and an increase in corneal sensitivity in $64.2 \%$ of cases. ${ }^{26}$ The study demonstrated that serum harbors neurotrophins and growth factors to the ocular surface. Other more recent studies confirmed that autologous serum eye drops allowed high rates of corneal healing, and also the improvement of corneal nerve morphology with increased number, length, width, and density detected by IVCM. ${ }^{27-29}$

The use of autologous serum may be inconvenient or contraindicated in patients with coexisting general conditions, such as anemia and blood discrasia. Therefore, the use of allogeneic sera obtained from healthy donors has been proposed as a viable alternative. Among these, umbilical cord blood serum is rich of epitheliotrophic growth factors, with higher levels of epidermal growth factor, insulin-like growth factor, transforming growth factor-beta, and vascular endothelial growth factors compared to peripheral blood serum. ${ }^{30,31}$ It has been shown that umbilical cord blood serum eye drops is effective in treating $\mathrm{NK}$, achieving a complete corneal healing in all the patients under study. ${ }^{32} \mathrm{~A}$ recent IVCM study showed an improvement in corneal nerve morphology in patients treated with umbilical cord serum eye drops, with an increase of total nerve number and a decrease of nerve tortuosity. ${ }^{33}$ Two randomized clinical trials are currently ongoing to evaluate safety and efficacy of topical umbilical cord blood serum and plasma rich in growth factors in patients with NK (NCT02707120, NCT03084861).

Surgical treatment is usually limited to corneal ulcers (stage 3) not responding to medical treatment, and/or to the related complications. Several approaches are available, including tarsorraphy, conjunctival flap, and amniotic membrane transplantation. Tarsorraphy is the most commonly used procedure to promote corneal healing in NK. It reduces the width of the palpebral fissure, protecting the cornea from the traumatic rubbing of eyelids and decreasing tear evaporation rate. ${ }^{34}$ However, the poor cosmetic outcome is often a major concern for patients. The injection of Botulin A toxin into the superior elevator palpebrae superioris muscle is an effective alternative, allowing also an easier and better examination of the ocular surface compared to tarsorraphy. ${ }^{35}$

The use of conjunctival flaps was first proposed by Gundersen in 1958 and became a standard surgical procedure to treat corneal ulcers with or without perforation. ${ }^{36}$ After a $360^{\circ}$ peritomy, a pedunculated conjunctival flap is mobilized from the upper bulbar conjunctiva and sutured to the conjunctiva in the lower limbus to cover the affected cornea. The conjunctival flap provides metabolic and mechanical support for corneal healing, thanks to the conjunctival blood vessels, which transport nutrients and growth factors to the corneal surface. ${ }^{37}$ The procedure restores the anatomical integrity of the ocular surface but sacrifices visual function.

Amniotic membrane transplantation has been successfully used to treat corneal ulcers and persistent epithelial defects from different causes, including NK. ${ }^{38,39}$ The amniotic membrane provides mechanical protection, releases growth factors, and supports epithelial cell adhesion and proliferation. ${ }^{38}$ Evidence from a randomized controlled trial showed that both amniotic membrane transplantation and conventional management (tarsorraphy or therapeutic contact lenses) are effective in treating refractive NK. ${ }^{40}$

Small perforations can be effectively treated with cyanoacrylate gluing followed by application of a bandage contact lens, whereas lamellar or penetrating keratoplasty is required for larger defects. ${ }^{41}$ However, persistent anesthesia causes impaired wound healing and a high risk of recurrence of corneal ulceration in the graft. This explains the low success rate of corneal transplantation in patients with NK.

Three cases from our cohort patients, which may be representative of different history and course of the disease, are shown in Figure 3. Explanation of each case is given in the legend.

\section{Future prospects}

NK was traditionally considered an orphan disease with no effective treatment. In recent years, novel promising medical and surgical treatments have been developed and are currently under investigation. Recent articles of novel medical treatments are summarized in Table 1. 

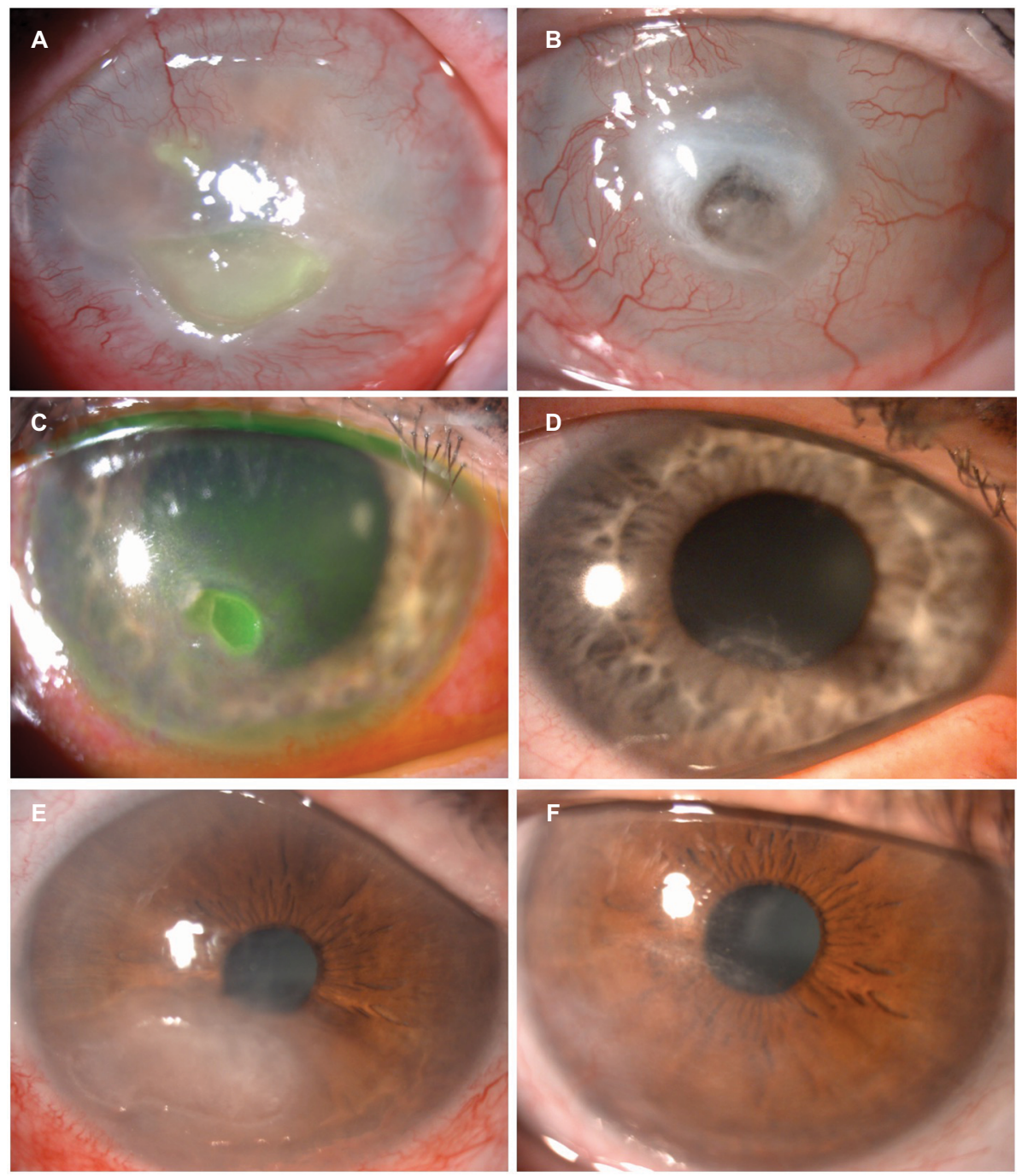

Figure 3 Slit-lamp pictures of a case of severe post-herpetic neurotrophic keratitis (case \#I, A and B).

Notes: A: Paracentral corneal neurotrophic ulcer with neovessels at the time of presentation. Preserved antibiotic and antiviral topical therapies were discontinued, while topical unpreserved tear substitutes and nocturnal ointments were started, in combination with oral antiviral therapy. B: After 8 months, the clinical picture worsened with impending central corneal perforation. Slit-lamp pictures of a case of neurotrophic keratitis secondary to trigeminal nerve damage from intracranial mass (case \#2, $\mathbf{C}$ and D). C: Small neurotrophic corneal ulcer in the inferior region of the cornea. Topical treatment with preservative-free tear substitutes and nocturnal ointments was started. D: After 6 months, the epithelial defect did not completely heal. Slit-lamp pictures of a corneal neurotrophic ulcer in a diabetic patient (case \#3, E and F). E: Large corneal neurotrophic ulcer in the inferotemporal region of the cornea. The patient was treated with topical preservative-free tear substitutes and nocturnal ointments. F: Three months after treatment, the ulcer completely healed.

Table I Novel medical treatments for neurotrophic keratitis

\begin{tabular}{|c|c|c|c|c|c|c|}
\hline Study & Eyes (no.) & Treatment & Posology & $\begin{array}{l}\text { Onset-treatment } \\
\text { interval (days) }\end{array}$ & $\begin{array}{l}\text { Complete } \\
\text { healing }\end{array}$ & $\begin{array}{l}\text { Healing } \\
\text { time (days) }\end{array}$ \\
\hline Aifa et $\mathrm{al}^{42}$ & 11 & RGTA & Ix/on alternate days & $>15$ & $72.7 \%$ & 60.9 \\
\hline Arvola et $\mathrm{al}^{43}$ & 6 & RGTA & Ix/on alternate days & 45 & $33 \%$ & 56 \\
\hline Dunn et $\mathrm{al}^{46}$ & 9 & Thymosin beta-4 & $4 x /$ day & $>42$ & $67 \%$ & 45 \\
\hline Nishida et $\mathrm{al}^{49}$ & 9 & SP and IGFI & $4 x /$ day & $|4|$ & $89 \%$ & 13.3 \\
\hline Yamada et $\mathrm{al}^{50}$ & 26 & SP and IGFI & $4 x /$ day & 96 & $73 \%$ & 10.5 \\
\hline Lambiase et $\mathrm{a}^{51}$ & 14 & NGF & Every $2 \mathrm{~h}$ for 2 days, then $6 \mathrm{x} /$ day & 45 & $100 \%$ & 21 \\
\hline Bonini et $\mathrm{a}^{2}$ & 45 & NGF & Every $2 \mathrm{~h}$ for 2 days, then $6 \mathrm{x} /$ day & 38 & $100 \%$ & $22.8 / 26.6^{a}$ \\
\hline Lee et $\mathrm{al}^{54}$ & 27 & Nicergoline (oral) & $10 \mathrm{mg} 2 x /$ day & $>60$ & $85 \%$ & 15.6 \\
\hline
\end{tabular}

Note: aRange (minimum/maximum).

Abbreviations: RGTA, regenerating agent; SP, substance P; IGF, insulin-like growth factor; NGF nerve growth factor; h, hours. 
Regenerating agent (RGTA, Cacicol20 ${ }^{\circledR}$ OTR3, Paris, France) is a new matrix agent containing large polymers mimicking heparan sulfates. RGTA creates a microenvironment that induces cell migration and adhesion and promotes epithelial healing. A Phase III clinical trial is currently ongoing (NCT01794312). In a recent study, topical drops containing RGTA achieved complete healing of corneal ulcer in $73 \%$ of 11 patients with NK. ${ }^{42}$ Another work showed less favorable results, with a failure of corneal healing in $67 \%$ of treated patients. ${ }^{43}$ The combined therapy of RGTA and umbilical cord blood serum eye drops has been recently proposed, thanks to their synergistic effect. ${ }^{44}$ RGTA could replace destroyed heparan sulfates, protecting the bioavailability of growth factors and providing a matrix in which cells can migrate and multiply. ${ }^{45}$

Thymosin beta-4 is an intracellular protein with various functions in different cellular processes, including wound healing, suppression of inflammation and apoptosis, synaptogenesis, and axon growth. ${ }^{46}$ The efficacy of topical treatment with thymosin beta-4 in nine patients with NK was described in an open study that reported a complete corneal healing in six patients with corneal ulcers, while the remaining three patients with punctuate epithelial defects did not show significant clinical changes ${ }^{46} \mathrm{~A}$ randomized-controlled trial is currently ongoing to evaluate safety and efficacy of topical treatment with thymosin beta-4 in patients with NK (NCT02600429).

Topical substance P (SP) and insulin-like growth factor 1 (IGF-1) stimulate synergistically the proliferation and migration of corneal epithelial cells both in vitro and in vivo. ${ }^{47,48} \mathrm{In}$ two recent studies, patients with epithelial defects associated to NK were treated with eye drops containing a combination of an SP-derived peptide and IGF-1-derived peptide. ${ }^{49,50}$ The treatment induced the healing of the epithelial defects in $89 \%{ }^{49}$ and $73 \%$ of cases $^{50}$ without any adverse side effect.

Topical treatment with NGF represents one of the most promising therapies for NK. NGF is a neurotrophin that promotes growth and survival of sensory and sympathetic neurons and can restore the function of injured neurons. ${ }^{9}$ NGF demonstrated a key role in the immune modulation, trophism, and healing of the ocular surface. ${ }^{11}$ Two open-label studies tested murine-derived NGF in patients with moderate (stage 2) and severe (stage 3) NK. The treatment induced rapid corneal healing in all the patients, with improved corneal sensitivity, tear production, and visual acuity without any adverse event. ${ }^{2,51}$ A recombinant human NGF eye drop formulation has been subsequently developed, and Phase I studies showed a good safety profile.$^{52}$ Phase II trials are currently ongoing to evaluate the efficacy of two different formulations of this treatment in patients with NK stage 2 and 3 (NCT02227147, NCT01756456).

Nicergoline is an ergoline derivative used to treat degenerative and vascular dementia. In animal rat models, it was shown to promote corneal healing and to increase NGF levels in the cornea. ${ }^{53} \mathrm{~A}$ recent study evaluated treatment with oral nicergoline in patients with $\mathrm{NK}$, demonstrating a healing of the epithelial defect in 23 out of 27 eyes, with an improvement of NGF levels and corneal sensitivity. ${ }^{54}$

Corneal neurotization is a novel and promising surgical procedure for NK, which can be performed through two different techniques, based on the transposition of the supraorbital and/or supratrochlear nerves (direct neurotization), ${ }^{55,56}$ or on the sural nerve graft (indirect neurotization). ${ }^{57}$ In the first technique, contralateral healthy supraorbital and supratrochlear nerves are harvested through a large coronal incision, tunneled across the bridge of the nose and inserted around the limbus of the anesthetic eye. Six patients with NK were treated with this technique, and all the patients showed the improvement of corneal health and sensitivity. ${ }^{55}$ Allevi et al performed corneal neurotization according to the same technique, combined with upper eyelid neurotized platysma graft, and followed by penetrating keratoplasty in a patient with associated seventh and fifth cranial nerve palsy. The overall procedure was successful allowing patient to regain corneal sensitivity, eyelid movement, and visual acuity. ${ }^{56} \mathrm{As}$ the ipsilateral frontal nerve is still intact in case of isolated corneal anesthesia from local damage to the long ciliary nerves, ipsilateral supraorbital nerve can be used to directly neurotize the cornea, allowing the decrease of the size of the scalp incision and the extent of the dissection needed. ${ }^{58}$

In the indirect technique, corneal neurotization was performed using a sural nerve autograft anastomosed to the supratrochlear nerve and tunneled through the upper eyelid incision. Unlike the direct technique, this approach enables the management of bilateral NK and avoids the large bicoronal incision. Four eyes with NK were included in the study: three of them had noticeably improved corneal sensitivity 6 months after surgery. ${ }^{57} \mathrm{~A}$ recent cadaver feasibility study described a new minimally invasive technique for corneal neurotization using an endoscopic approach for direct nerve supraorbital nerve transfer..$^{59}$ The potential advantages of the endoscopic corneal neurotization include smaller incisions, decreased operative and healing time, decreased forehead edema, less risk for facial nerve injury, and minimal blood loss. A pilot study is currently ongoing for NK stages 2 and 3 (NCT03037450). 


\section{Acknowledgment}

The patients gave their consent for the photographs to be used in medical publication.

\section{Disclosure}

The authors report no conflicts of interest in this work.

\section{References}

1. Mackie IA. Neuroparalytic keratitis. In: Fraunfelder F, Roy FH, Meyer SM, editors. Current Ocular Therapy. Philadelphia, PA, USA:WB Saunders; 1995.

2. Bonini S, Lambiase A, Rama P, Caprioglio G, Aloe L. Topical treatment with nerve growth factor for neurotrophic keratitis. Ophthalmology. 2000;107(7):1347-1351.

3. Hsu HY, Modi D. Etiologies, quantitative hypoesthesia, and clinical outcomes of neurotrophic keratopathy. Eye Contact Lens. 2015;41(5):314-317.

4. Muller LJ, Marfurt CF, Kruse F, Tervo TM. Corneal nerves: structure, contents and function. Exp Eye Res. 2003;76(5):521-542.

5. Rozsa AJ, Beuerman RW. Density and organization of free nerve endings in the corneal epithelium of the rabbit. Pain. 1982;14(2):105-120.

6. Sigelman S, Friedenwald JS. Mitotic and wound-healing activities of the corneal epithelium: effect of sensory denervation. AMA Arch Ophthalmol. 1954;52(1):46-57.

7. Alper MG. The anhestetic eye: an investigation of changes in the anterior ocular segment of the monkey caused by interrupting the trigeminal nerve at various levels along its course. Trans Am Ophthalmol Soc. 1975;73:323-365.

8. Heigle TJ, Pflugfelder SC. Aqueous tear production in patients with neurotrophic keratitis. Cornea. 1996;15(2):135-138.

9. Levi-Montalcini R. The nerve growth factor 35 years later. Science. 1987;237(4819):1154-1162.

10. Lambiase A, Manni L, Bonini S, Rama P, Micera A, Aloe L. Nerve growth factor promotes corneal healing: structural, biochemical, and molecular analyses of rat and human corneas. Invest Ophthalmol Vis Sci. 2000;41(5):1063-1069.

11. Bonini S, Aloe L, Bonini S, Rama P, Lamagna A, Lambiase A. Nerve growth factor (NGF): an important molecule for trophism and healing of the ocular surface. Adv Exp Med Biol. 2002;506(Pt A):531-537.

12. Sacchetti M, Lambiase A. Diagnosis and management of neurotrophic keratitis. Clin Ophthalmol. 2014;8:571-579.

13. Semeraro F, Forbice E, Romano V, et al. Neurotrophic keratitis. Ophthalmologica. 2014;231(4):191-197.

14. Hamrah P, Cruzat A, Dastjerdi MH, et al. Corneal sensation and sub-basal nerve alterations in patients with herpes simplex keratitis: an in vivo confocal microscopy study. Ophthalmology. 2010;117(10):1930-1936.

15. Lee BH, McLaren JW, Erie JC, Hodge DO, Bourne WM. Reinnervation in the cornea after LASIK. Invest Ophthalmol Vis Sci. 2002;43(12):3660-3664.

16. Lambiase A, Sacchetti M, Mastropasqua A, Bonini S. Corneal changes in neurosurgically induced neurotrophic keratitis. JAMA Ophthalmol. 2013;131(12):1547-1553.

17. Dhillon VK, Elalfy MS, Al-Aqaba M, Gupta A, Basu S, Dua HS. Corneal hypoesthesia with normal sub-basal nerve density following surgery for trigeminal neuralgia. Acta Ophthalmol. 2016;94(1): e6-e10.

18. Rosenberg ME, Tervo TM, Immonen IJ, Muller LJ, Gronhagen-Riska $\mathrm{C}$, Vesaluoma MH. Corneal structure and sensitivity in type 1 diabetes mellitus. Invest Ophthalmol Vis Sci. 2000;41(10):2915-2921.

19. Tavakoli M, Marshall A, Pitceathly R, et al. Corneal confocal microscopy: a novel means to detect nerve fibre damage in idiopathic small fibre neuropathy. Exp Neurol. 2010;223(1):245-250.
20. Jabbarvand M, Hashemian H, Khodaparast M, Rafatnejad A, Beheshtnejad A, Salami A. Do unilateral herpetic stromal keratitis and neurotrophic ulcers cause bilateral dry eye? Cornea. 2015;34(7):768-772.

21. Norn MS. Measurement of sensitivity. In: Norn MS, editor. External Eye Diseases. Methods of Examination. Copenhagen, Denmark: Munksgaard International Publisher Ltd; 1974.

22. Belmonte C, Acosta MC, Schmelz M, Gallar J. Measurement of corneal sensitivity to mechanical and chemical stimulation with a $\mathrm{CO} 2$ esthesiometer. Invest Ophthalmol Vis Sci. 1999;40(2):513-519.

23. Grey F, Carley F, Biswas S, Tromans C. Scleral contact lens management of bilateral exposure and neurotrophic keratopathy. Cont Lens Anterior Eye. 2012;35(6):288-291

24. Giannaccare G, Versura P, Buzzi M, Primavera L, Pellegrini M, Campos EC. Blood derived eye drops for the treatment of cornea and ocular surface diseases. Transfus Apher Sci. 2017;56(4):595-604.

25. Bradley JC, Bradley RH, Mccartney DL, Mannis MJ. Serum growth factor analysis in dry eye syndrome. Clin Exp Ophthalmol. 2008;36(8):717-720.

26. Matsumoto Y, Dogru M, Goto E, et al. Autologous serum application in the treatment of neurotrophic keratopathy. Ophthalmology. 2004;111(6): $1115-1120$.

27. Guadilla AM, Balado P, Baeza A, Merino M. [Effectiveness of topical autologous serum treatment in neurotrophic keratopathy]. Arch Soc Esp Oftalmol. 2013;88(8):302-306. Spanish.

28. Turkoglu E, Celik E, Alagoz G. A comparison of the efficacy of autologous serum eye drops with amniotic membrane transplantation in neurotrophic keratitis. Semin Ophthalmol. 2014;29(3):119-126.

29. Rao K, Leveque C, Pflugfelder SC. Corneal nerve regeneration in neurotrophic keratopathy following autologous plasma therapy. $\mathrm{Br} J$ Ophthalmol. 2010;94(5):584-591.

30. Versura P, Buzzi M, Giannaccare G, et al. Targeting growth factor supply in keratopathy treatment: comparison between maternal peripheral blood and cord blood as sources for the preparation of topical eye drops. Blood Transfus. 2016;14(2):145-151.

31. Versura P, Buzzi M, Giannaccare G, et al. Cord blood serum-based eye drops: the impact of donor haematological and obstretic factors on the variability of epidermal growth factor levels. Blood Transfus. 2014;12(Suppl 1):S44-S50.

32. Yoon KC, You IC, Im SK, Jeong TS, Park YG, Choi J. Application of umbilical cord serum eyedrops for the treatment of neurotrophic keratitis. Ophthalmology. 2007;114(9):1637-1642.

33. Giannaccare G, Buzzi M, Fresina M, Velati C, Versura P. Efficacy of 2-month treatment with cord blood serum eye drops in ocular surface disease: an in vivo confocal miscroscopy study. Cornea. 2017;36(8):915-921.

34. Cosar CB, Cohen EJ, Rapuano CJ, et al. Tarsorrhaphy clinical experience from a cornea practice. Cornea. 2001;20(8):789-791.

35. Kirkness CM, Adams GG, Dilly PN, Lee JP. Botulinum toxin A-induced protective ptosis in corneal disease. Ophthalmology. 1988;95(5):473-480.

36. Gundersen T. Conjunctival flaps in the treatment of corneal disease with reference to a new technique of application. AMA Arch Ophthalmol. 1958;60(5):880-888.

37. Khodadoust A, Quinter AP. Microsurgical approach to the conjunctival flap. Arch Ophthalmol. 2003;121(8):1189-1193.

38. Chen HJ, Pires RT, Tseng SC. Amniotic membrane transplantation for severe neurotrophic corneal ulcers. Br J Ophthalmol. 2000;84(8): 826-833.

39. Lee SH, Tseng SC. Amniotic membrane transplantation for persistent epithelial defects with ulceration. Am J Ophthalmol. 1997;123(3): 303-312.

40. Khokhar S, Natung T, Sony P, Sharma N, Agarwal N, Vajpayee RB. Amniotic membrane transplantation in refractory neurotrophic corneal ulcers: a randomized, controlled clinical trial. Cornea. 2005;24(4):654-660. 
41. Fogle JA, Kenyon KR, Foster CS. Tissue adhesive arrests stromal melting in the human cornea. Am J Ophthalmol. 1980;89(6): 795-802.

42. Aifa A, Gueudry J, Portmann A, Delcampe A, Muraine M. Topical treatment with a new matrix therapy agent (RGTA) for the treatment of corneal neurotrophic ulcers. Invest Ophthalmol Vis Sci. 2012;53(13): 8181-8185.

43. Arvola RP, Robciuc A, Holopainen JM. Matrix regeneration therapy: a case series of corneal neurotrophic ulcers. Cornea. 2016;35(4):451-455.

44. Giannaccare G, Fresina M, Vagge A, Versura P. Synergistic effect of regenerating agent plus cord blood serum eye drops for the treatment of resistant neurotrophic keratitis: a case report and a hypothesis for pathophysiologic mechanism. Int Med Case Rep J. 2015;8:277-281.

45. Rouet V, Hamma-Kourbali Y, Petit E, et al. A synthetic glycosaminoglycan mimetic bind vascular endothelial growth factor and modulates angiogenesis. J Biol Chem. 2005;280(38):32792-32800.

46. Dunn SP, Heidemann DG, Chow CY, et al. Treatment of chronic nonhealing neurotrophic corneal epithelial defects with thymosin beta4. Ann NY Acad Sci. 2010;1194:199-206.

47. Nishida T, Nakamura M, Ofuji K, Reid TW, Mannis MJ, Murphy CJ. Synergistic effects of substance $\mathrm{P}$ with insulin-like growth factor-1 on epithelial migration of the cornea. J Cell Physiol. 1996;169(1): 159-166.

48. Nakamura M, Ofuji K, Chikama T, Nishida T. Combined effects of substance $\mathrm{P}$ and insulin-like growth factor-1 on corneal epithelial wound closure of rabbit in vivo. Curr Eye Res. 1997;16(3):275-278.

49. Nishida T, Chikama T, Morishige N, Yanai R, Yamada N, Saito J. Persistent epithelial defects due to neurotrophic keratopathy treated with a substance p-derived peptide and insulin-like growth factor 1. Jpn J Ophthalmol. 2007;51(6):442-447.
50. Yamada N, Matsuda R, Morishige N, et al. Open clinical study of eye-drops containing tetrapeptides derived from substance $\mathrm{P}$ and insulin-like growth factor-1 for treatment of persistent corneal epithelial defects associated with neurotrophic keratopathy. Br J Ophthalmol. 2008;92(7):896-900.

51. Lambiase A, Rama P, Bonini S, Caprioglio G, Aloe L. Topical treatment with nerve growth factor for corneal neurotrophic ulcers. NEngl J Med. 1998;338(17):1174-1180.

52. Ferrari MP, Mantelli F, Sacchetti M, et al. Safety and pharmacokinetics of escalating doses of human recombinant nerve growth factor eye drops in a double-masked, randomized clinical trial. BioDrugs. 2014;28(3):275-283.

53. Kim SY, Choi JS, Joo CK. Effects of nicergoline on corneal epithelial wound healing in rat eyes. Invest Ophthalmol Vis Sci. 2009;50(2):621-625.

54. Lee YC, Kim SY. Treatment of neurotrophic keratopathy with nicergoline. Cornea. 2015;34(3):303-307.

55. Terzis JK, Dryer MM, Bodner BI. Corneal neurotization: a novel solution to neurotrophic keratopathy. Plast Reconstr Surg. 2009;123(1):112-120.

56. Allevi F, Fogagnolo P, Rossetti L, Biglioli F. Eyelid reanimation, neurotisation, and transplantation of the cornea in a patient with facial palsy. BMJ Case Rep. 2014:pii: bcr2014205372.

57. Elbaz U, Bains R, Zuker RM, Borschel GH, Ali A. Restoration of corneal sensation with regional nerve transfers and nerve grafts: a new approach to a difficult problem. JAMA Ophthalmol. 2014;132(11):1289-1295.

58. Jacinto F, Espana E, Padilla M, Ahmad A, Leyngold I. Ipsilateral supraorbital nerve transfer in a case of recalcitrant neurotrophic keratopathy with an intact ipsilateral frontal nerve: a novel surgical technique. $\mathrm{Am}$ J Ophthalmol Case Rep. 2016;4:14-17.

59. Leyngold I, Weller C, Leyngold M, et al. Endoscopic corneal neurotization: Cadaver Feasibility Study. Ophthal Plast Reconstr Surg. Epub 2017 May 2.
Eye and Brain

\section{Publish your work in this journal}

Eye and Brain is an international, peer-reviewed, open access journal focusing on clinical and experimental research in the field of neuroophthalmology. All aspects of patient care are addressed within the journal as well as basic research. Papers covering original research, basic science, clinical and epidemiological studies, reviews and evaluations,

\section{Dovepress}

guidelines, expert opinion and commentary, case reports and extended reports are welcome. The manuscript management system is completely online and includes a very quick and fair peer-review system, which is all easy to use. Visit http://www.dovepress.com/testimonials.php to read real quotes from published authors. 